01

\title{
Соударения медленных электронов с молекулами тимина
}

\author{
(С) И.В. Чернышова, Е.Э. Контрош, О.Б. Шпеник
}

Институт электронной фризики НАН Украины, 88017 Ужгород, Украина

e-mail: irinav.chernyshova@gmail.com

Поступила в редакцию 14.06.2018 г.

В окончательной редакции 10.09.2018 г.

С использованием гипоциклоидального электронного спектрометра измерены полное сечение рассеяния медленных $(0-9 \mathrm{eV})$ электронов и сечение диссоциативного прилипания электронов к молекулам тимина в газовой фазе, а также проведены исследования сечения ионизации молекулы в области энергий $9-32 \mathrm{eV}$. В сечении рассеяния обнаружены особенности, обусловленные образованием и распадом короткоживущих состояний отрицательного иона молекулы. Три из них $(E=0.32,1.71$ и $4.03 \mathrm{eV})$ относятся к резонансам формы, остальные, наблюдаемые нами впервые - к резонансам Фешбаха (или к core-ехсіted резонансам). В полном сечении диссоциативного прилипания в области энергий $E<4 \mathrm{eV}$ наблюдается четкая структура, обусловленная образованием отрицательного иона $(T-H)^{-}$, а выше $4 \mathrm{eV}-$ менее интенсивная структура, связанная с суммарным вкладом фрагментных ионов тимина. Проанализирована корреляция особенностей, обнаруженных в полном сечении рассеяния и в сечении диссоциативного прилипания. Абсолютное полное сечение рассеяния получено нормировкой измеренной кривой на теоретический расчет. В полном сечении ионизации наблюдаются особенности, связанные как с влиянием образования фрагментных ионов, так и с ионизацией вследствие выбивания электронов с орбиталей внешней оболочки молекулы.

DOI: $10.21883 / O S .2019 .02 .47190 .162-18$

\section{Введение}

Взаимодействие низкоэнергетических (медленных) электронов с молекулами ДНК, РНК и их компонентами в последнее время является предметом интенсивных исследований $[1,2]$. Эксперименты [3-5] показали, что медленные электроны $(E<15 \mathrm{eV})$ могут приводить к разрыву одной или двух нитей ДНК и, следовательно, к повреждениям макро- и микроструктуры этих молекул, вызывающих гибель отдельных клеток и организма в целом. Это открытие послужило стимулом для ряда экспериментов, целью которых являлось объяснение различных аспектов взаимодействия медленных электронов с молекулами ДНК и РНК.

В экспериментах [6-20], выполненных с парообразными (газообразными) мишенями, исследовались соударения электронов с молекулярными подгруппами, включающими пуриновые (аденин (A) и гуанин $(\mathrm{G})$ ), а также пиримидиновые (цитозин (С) и тимин $(\mathrm{T})$ ) основания ДНК и урацил $(\mathrm{U})$ - основание РНК. В большинстве из этих экспериментов изучалось диссоциативное прилипание [7,9-16] и/или возбуждение электронным ударом и ионизация $[8,11,14,16-20]$. Только авторы работы [6] выполнили трансмиссионные эксперименты, в которых определили энергии резонансов в сечениях рассеяния электронов молекулами U, T, C, G и А и, основываясь на своих расчетах, идентифицировали особенности, наблюдаемые в сечениях рассеяния, как $\pi^{*}$-резонансы формы. Спектры энергетических потерь электронов были измерены авторами [8] для изучения электронного и колебательного возбуждений молекулы тимина. Диссоциативное прилипание электронов к молекулам А, C, Т исследовалось в работах $[13,15]$. В своих измерениях авторы [13] использовали экспериментальную установку с пересекающимися (электронным и нейтральным молекулярным) пучками в сочетании с квадрупольным масс-спектрометром. Кроме наблюдаемых ранее ионов $(\mathrm{C}-\mathrm{H})^{-}$и $(\mathrm{T}-\mathrm{H})^{-}$, авторы детектировали пять других анионных фрагментов для молекулы С и восемь для Т и изучили их выход в зависимости от энергии бомбардирующих электронов.

В работе [15] для исследования процесса диссоциативного прилипания применялась трансмиссионная спектроскопия с модифицированным трохоидальным электронным спектрометром, который ранее использовался для определения вертикальных энергий прилипания [6]. Для селекции электронов в пучке по энергии применялось магнитное поле. Ток отрицательных ионов, образованных в соударениях электронов с молекулами, детектировался на стенки ячейки столкновений. Значение сечения определялось из соотношения тока отрицательных ионов, детектируемого при низких энергиях электронного пучка, и тока положительных ионов, определенного в максимуме сечения ионизации. Абсолютное значение сечения диссоциативного прилипания получали нормировкой на сечение образования положительных ионов, рассчитанное с использованием приближения binary-encounter-Bethe (BEB) [21,22].

Для молекул азотистых оснований нуклеиновых кислот имеются как экспериментальные, так и теоретические данные по ионизации электронным ударом $[11,23,24]$ и фотонами [25-27]. Такие исследования позволяют получить данные об адиабатических и вертикальных потенциалах ионизации (IP), а также об энер- 
гиях появления (AЕ) различных ионных фрагментов, образующихся при диссоциации молекулярных ионов. Масс-спектрометрические исследования фрагментации молекулы тимина в процессе ионизации выполнены в работе [23] при двух значениях энергии ионизирующих электронов - 70 и $20 \mathrm{eV}$. Измерения, выполненные при энергии электронов $70 \mathrm{eV}$, дают более богатый масс-спектр, чем при меньших энергиях электронов. В масс-спектр, измеренный при энергии ионизирующих электронов $20 \mathrm{eV}$, доминирующий вклад вносит материнский ион $\mathrm{C}_{5} \mathrm{H}_{6} \mathrm{~N}_{2} \mathrm{O}_{2}^{+}$с массовым числом $m / z=126 \mathrm{amu}$ (atomic mass unit) и, кроме того, наблюдаются ионные фрагменты с $m / z=83$ и $55 \mathrm{amu}$.

Для молекулы тимина также выполнены эксперименты с высокоэнергетическими электронами с целью определения энергии связи молекулярных орбиталей [28]. Найдены энергии связи 9 орбиталей внешней оболочки молекулы. В работе [26] с использованием метода многочастичных функций Грина рассчитаны вертикальные энергии ионизации 14 орбиталей внешней оболочки молекулы.

В литературе, однако, приводятся противоречивые данные как об абсолютных значениях сечений образования отрицательных и положительных ионов молекул азотистых оснований нуклеиновых кислот, так и о порогах появления молекулярных ионов и их фрагментов, поэтому постановка новых прецизионных исследований является актуальной и необходимой. В предыдущих исследованиях $[29,30]$ мы изучали процессы возбуждения молекул азотистых оснований нуклеиновых кислот урацила и тимина в газовой фазе медленными электронами. Настоящая работа посвящена исследованию взаимодействия медленных электронов с молекулами тимина, а именно процессов рассеяния электронов и образования отрицательных и положительных ионов.

\section{Эксперимент и методика}

Для исследования соударений электронов с молекулами тимина в газовой фазе использовался гипоциклоидальный электронный спектрометр [31], состоящий из двух идентичных гипоциклоидальных электронных монохроматоров (ГЭМ) [32], размещенных последовательно: один из них служил монохроматором электронов, а другой - анализатором рассеянных электронов. Между ними располагалась паронаполненная ячейка, служащая камерой столкновений. Выбор данного спектрометра обусловлен его уникальностью, заключающейся в том, что он может работать в области очень малых (практически нулевых) значений энергии, обладая при этом высокой степенью пропускания (до 95\%). В данных измерениях наилучшее энергетическое разрешение ГЭМ (полная ширина на полувысоте максимума продифференцированного начального участка вольт-амперной характеристики электронного тока) составляло $\Delta E_{1 / 2}=80 \mathrm{meV}$ при токе электронов $120 \mathrm{nA}$.
Весь спектрометр помещался в однородное магнитное поле напряженностью 120 Ое, создаваемое парой колец Гельмгольца (с внутренним диаметром $210 \mathrm{~mm}$ ), вдоль силовых линий магнитного поля.

Мишень в газовой фазе формировалась в резервуаре молекулярного источника, который представлял собой полый цилиндр из нержавеющей стали, резистивно нагреваемый до температуры, при которой еще не наблюдаются структурные изменения молекулы. Температуры резервуара и ячейки контролировались термопарами.

Порошок тимина, помещенный в кварцевую ампулу, находящуюся в резервуаре молекулярного источника, постепенно нагревался до температуры $120^{\circ} \mathrm{C}$. Пары тимина по металлической трубке поступали непосредственно в паронаполненную ячейку. Отметим, что для предотвращения конденсации молекул тимина на электродах спектрометра его температура поддерживалась на $20-30^{\circ} \mathrm{C}$ выше температуры резервуара с исследуемым веществом. Рабочее давление в вакуумной камере было не больше $2 \cdot 10^{-6} \mathrm{~Pa}$.

При изучении полного сечения рассеяния (ПСР) электронов нами был использован традиционный трансмиссионный метод, в котором измерялось ослабление электронного пучка при его прохождении через пары исследуемых молекул и с использованием формулы Ламберта-Бера определялось сечение рассеяния:

$$
\sigma(E)=(l / n) \ln \left[I_{0}(E) / I(E)\right]
$$

где $I(E)$ и $I_{0}(E)$ - интенсивности электронного пучка при наличии и в отсутствие паров мишени в камере столкновений длиной $l(\mathrm{~mm})$ соответственно, $n-$ плотность паров мишени.

При изучении сечения образования отрицательных (положительных) ионов ток ионов, образовавшихся при соударении электронов с молекулами тимина, детектировался на коллектор ионов, установленный внутри камеры столкновений перпендикулярно к направлению электронного пучка. Перед коллектором размещалась сетка (прозрачностью 80\% из немагнитного материала) для его защиты от попадания вторичных и рассеянных электронов. Для регистрации отрицательных ионов на коллектор подавался небольшой положительный потенциал, а при регистрации положительных ионов небольшой отрицательный потенциал относительно камеры столкновений.

\section{Результаты измерений и их обсуждение}

Молекула тимина $\left(\mathrm{C}_{5} \mathrm{H}_{6} \mathrm{~N}_{2} \mathrm{O}_{2}\right)$ - производное пиримидина (имеет в своем составе одно шестичленное пиримидиновое кольцо), является одним из четырех нуклеотидных оснований, важным компонентом макромолекул ДНК. В наших экспериментах использовался образец тимина производства компании Sigma-Aldrich в виде белого кристаллического порошка (чистотой 99\%) 
без дополнительной очистки. Температура плавления тимина $316^{\circ} \mathrm{C}$ [33].

\section{Рассеяние электронов молекулами}

Энергетическая зависимость полного сечения рассеяния электронов молекулами тимина, измеренная в области энергий электронов от 0 до $9.50 \mathrm{eV}$, приведена на рис. 1. Отличительной особенностью измеренного нами сечения является его сильный спад с увеличением энергии электронов от 0 до $1 \mathrm{eV}$, что характерно для полярных молекул [34,35]. В этой области энергий $(E<1 \mathrm{eV})$ на сильном спаде сечения наблюдается слабовыраженная особенность. В области энергий $1-5 \mathrm{eV}$ наблюдаются две четкие резонансные особенности, а выше $5.00 \mathrm{eV}$ можно выделить еще несколько слабо выраженных особенностей.

Наиболее выраженные особенности в ПСР электронов молекулами тимина (при энергиях $1.71 \mathrm{u} 4.03 \mathrm{eV}$ ) обусловлены влиянием резонансов формы, которые образуются при кратковременном $\left(10^{-15} \mathrm{~s}\right)$ захвате электрона молекулой на свободную $\pi^{*}$-орбиталь. Эти резонансы ранее были обнаружены в [6] и идентифицированы как антисвязанные состояния. На рис. 1 вертикальными линиями, обозначенными $\pi_{1}^{*}-\pi_{3}^{*}$, показаны положения этих резонансов [6]. Для выделения резонансных особенностей, обнаруженных в ПСР, и более точного определения их энергетического положения мы вычли из измеренной энергетической зависимости сечения $\left(\sigma_{t}\right)$ аппроксимированную гладкую кривую подложки сечения рассеяния $\left(\sigma_{\text {app }}\right)$. Выделенные резонансные вклады $\left(\sigma_{t}-\sigma_{\text {app }}\right)$ показаны на вставке рис. 1 . Положения особенностей $(E)$ в $\mathrm{eV}$ приведены в табл. 1, где для сравнения также представлены данные экспериментов [6] и теоретических расчетов $[6,16,36,37]$. Как видно из рис. 1 и табл. 1 , положения наблюдаемых нами особенностей хорошо согласуются с данными работы [6].

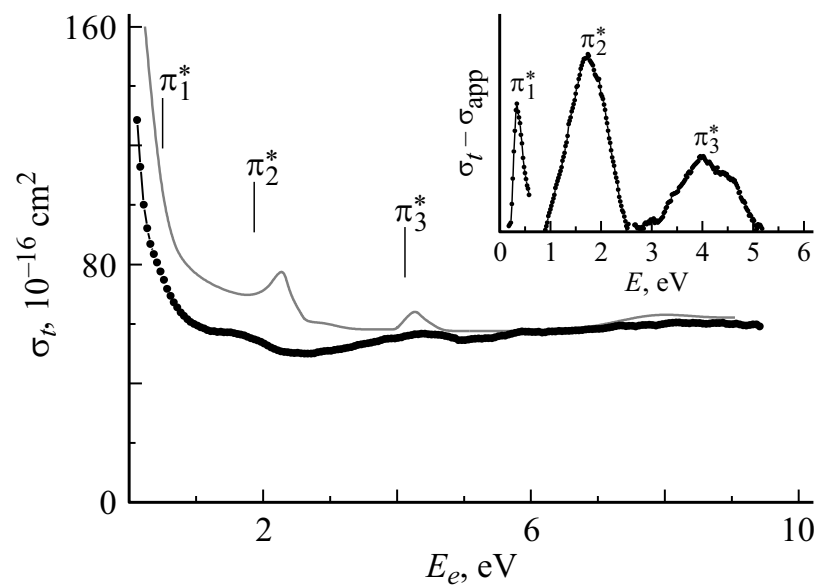

Рис. 1. Энергетическая зависимость полного сечения рассеяния электронов молекулами тимина (точки - наши результаты, сплошная линия - расчет [37]). На вставке показан резонансный вклад в сечение рассеяния.

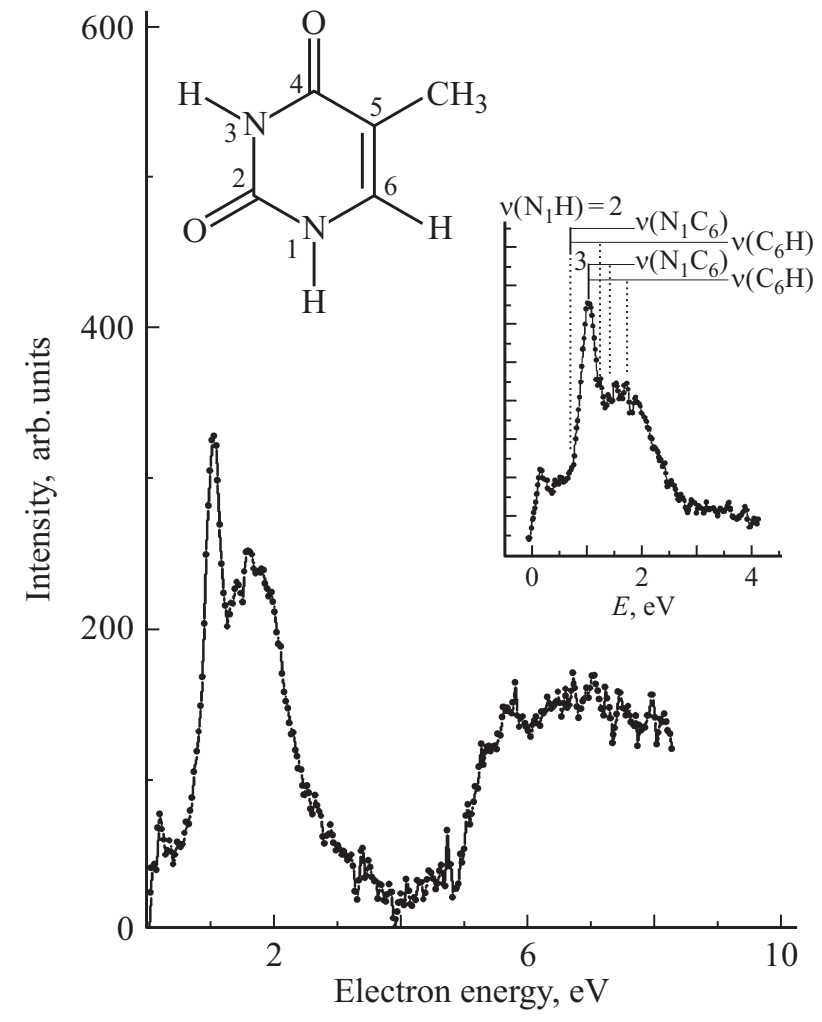

Рис. 2. Энергетическая зависимость полного сечения диссоциативного прилипания электронов к молекулам тимина. На вставке показано это сечение в области $0-4 \mathrm{eV}$, измеренное с меньшим шагом сканирования по энергии $(0.02 \mathrm{eV})$. Указаны колебательные уровни, заимствованные из работы [16].

Особенность, наблюдаемая в области энергий $3-5 \mathrm{eV}$ с максимумом при значении энергии $4.03 \mathrm{eV}$, носит сложный характер. По-видимому, она представляет собой результат перекрывания нескольких состояний. Мы воспользовались методом нелинейного фитирования лоренцевыми контурами для определения энергетического положения максимумов, наблюдаемых в этой области энергий (табл. 1). Расчеты [16] свидетельствуют о наличии низкоэнергетических $\sigma^{*}$-резонансов формы в этой области энергий $\left(\sigma_{2}^{*}=3.42 \mathrm{eV}\right.$ и $\left.\sigma_{3}^{*}=3.75 \mathrm{eV}\right)$. Энергетические положения этих резонансов хорошо coгласуются с результатами наших исследований (табл. 1).

Выше энергии $4.5 \mathrm{eV}$ наблюдаются особенности, которые, по-видимому, следует отнести к резонансам Фешбаха (или core-excited resonances) [38]. Отметим, что подобные резонансы наблюдались в расчетах, выполненных $R$-матричным методом для молекулы урацила [35], химически близкой к молекуле тимина. Основное отличие между ними состоит в наличии метильной группы при углероде С5 у тимина (см. структурную формулу молекулы на рис. 2).

Для определения абсолютного значения полного сечения рассеяния электронов молекулами тимина мы пронормировали наши данные при энергии $5.80 \mathrm{eV}$ на расчет [37]. Как видно из рис. 1, сечение рассеяния 
Таблица 1. Энергетическое положение особенностей, наблюдаемых в полном сечении рассеяния электронов на молекулах тимина

\begin{tabular}{|c|c|c|c|c|c|c|c|}
\hline \multicolumn{2}{|c|}{ Эксперимент } & \multicolumn{6}{|c|}{ Теория } \\
\hline $\begin{array}{c}\text { Наши данные } \\
( \pm 0.05 \mathrm{eV})\end{array}$ & {$[6]$} & {$[36]$} & & $\mathrm{eV})$ & [37] & & \\
\hline$E, \mathrm{eV}$ & $E, \mathrm{eV}$ & $E, \mathrm{eV}$ & $E, \mathrm{eV}$ & Состояние & $E, \mathrm{eV}$ & $E, \mathrm{eV}$ & Состояние \\
\hline $\begin{array}{l}0.32 \\
1.71 \\
2.57 \\
3.41 \\
3.75 \\
4.03 \\
4.55 \\
5.37 \\
6.82 \\
7.46 \\
8.28\end{array}$ & $\begin{array}{l}0.29 \\
1.71 \\
\\
4.05\end{array}$ & 0.32 & $\begin{array}{l}0.364 \\
1.771 \\
\\
4.057\end{array}$ & $\begin{array}{l}\pi_{1}^{*} \\
\pi_{2}^{*} \\
\\
\\
\pi_{3}^{*}\end{array}$ & $\begin{array}{l}0.3 \\
1.9 \\
\\
\\
5.7\end{array}$ & $\begin{array}{l}2.40 \\
3.42 \\
3.75\end{array}$ & $\begin{array}{l}\sigma_{1}^{*} \\
\sigma_{2}^{*} \\
\sigma_{3}^{*}\end{array}$ \\
\hline
\end{tabular}

достигает значения $60 \cdot 10^{-16} \mathrm{~cm}^{2}$ при этой энергии и до энергии $9.50 \mathrm{eV}$ практически не изменяется.

\section{Образование отрицательных ионов}

Образование отрицательных ионов при соударении электронов низких энергий с нейтральными молекулами является, как правило, резонансным процессом и осуществляется через диссоциативное прилипание электронов к молекулам [39]: на начальном этапе происходит образование короткоживущего состояния отрицательного иона молекулы, которое далее распадается на отрицательный ион и фрагменты.

На рис. 2 приведена измеренная нами энергетическая зависимость сечения диссоциативного прилипания электронов к молекуле тимина в области энергий бомбардирующих электронов 0-8.20 eV. Измерения выполнялись при токе первичного электронного пучка $20 \mathrm{nA}$, шаг сканирования по энергии составлял 20 и $50 \mathrm{meV}$, моноэнергетичность была $200 \mathrm{meV}$. Особое внимание уделялось области энергий $0-4 \mathrm{eV}$, где наблюдается интенсивная структура. Отметим, что измеряемое нами сечение диссоциативного прилипания имеет смысл полного сечения, поскольку измеряются вклады всех образованных в камере столкновений отрицательных ионов. Из рис. 2 видно, что на энергетической зависимости сечения диссоциативного прилипания наблюдаются две выраженные структуры: одна - в области энергий от 0 до $4 \mathrm{eV}$, вторая - от $4 \mathrm{eV}$ и выше. Интенсивность второй структуры приблизительно в два раза меньше интенсивности пика при значении энергии $1.02 \mathrm{eV}$. Видно, что образование отрицательных ионов тимина наиболее эффективно происходит в узкой области малых энергий $0-4 \mathrm{eV}$, носит резонансный характер. Здесь наблюдаются узкий интенсивный максимум при $1.02 \mathrm{eV}$ и более широкий, но менее интенсивный максимум при $\sim 1.73 \mathrm{eV}$.

На вставке рис. 2 представлены результаты измерений полного сечения диссоциативного прилипания в области энергий 0-4 eV, выполненные с меньшим шагом $(\Delta E=20 \mathrm{meV})$ сканирования по энергии. Это позволило лучше проявиться колебательной структуре молекулы. Показанные на вставке колебательные уровни представляют результаты расчетов, взятые из работы [16]. Положения особенностей, наблюдаемых на измеренной кривой, приводятся в табл. 2. Для их анализа мы воспользовались данными работы [13].

Как видно, наибольший вклад в сечение прилипания вносят ионные фрагменты $(\mathrm{T}-\mathrm{H})^{-}$. Они наблюдаются в области энергий ниже $4 \mathrm{eV}$ и образуются элиминированием атома водорода из материнского молекулярного аниона:

$$
e^{-}+\mathrm{T} \leftrightarrow(\mathrm{T})^{-*} \rightarrow(\mathrm{T}-\mathrm{H})^{-}+\mathrm{H}^{\bullet}
$$

В области энергий выше $4 \mathrm{eV}$ основной вклад происходит от следующих ионных фрагментов: $(\mathrm{T}-2 \mathrm{H})^{-}$, $\left(\mathrm{C}_{4} \mathrm{H}_{5} \mathrm{~N}_{2} \mathrm{O}\right)^{-},\left(\mathrm{C}_{3} \mathrm{H}_{2} \mathrm{NO}\right)^{-},\left(\mathrm{C}_{3} \mathrm{H}_{4} \mathrm{~N}\right)^{-},(\mathrm{OCN})^{-}$и $(\mathrm{CN})^{-}$.

Как было показано в [16], выход нейтральных атомов Н (дегидрирование) является избирательным к их положению в молекуле, и происходит исключительно из положений N1 и N3 (см. структурную формулу молекулы на рис. 2). Причем механизм дегидрирования из этих двух возможных положений различен. Так, потеря атома Н из положения N1 (связь N1-H) происходит через образование резонанса Фешбаха при колебательном возбуждении (vibrational Feshbach resonance) (подробнее см. в [16]). Это приводит к появлению максимумов (небольшого при энергии $0.7 \mathrm{eV}$ и интенсивного при $1.02 \mathrm{eV}$ ) в сечении (вставка на рис. 2). Из положения N3 атомы Н отрываются вследствие образования и распада промежуточного (короткоживущего) состояния 
Таблица 2. Сравнение энергетического положения особенностей, наблюдаемых в полном сечении диссоциативного прилипания электронов к молекулам тимина с положениями резонансов в выходе ионных фрагментов, сформированных в процессе диссоциативного прилипания электронов к молекулам тимина [13]

\begin{tabular}{|c|c|c|c|c|c|c|c|}
\hline \multirow{2}{*}{$\begin{array}{l}\text { Наши результаты } \\
(\text { в } \mathrm{eV})\end{array}$} & \multicolumn{7}{|c|}{$\begin{array}{c}\text { Энергетическое положение резонансов для выхода ионных фрагментов [13] } \\
\text { (в еV) }\end{array}$} \\
\hline & $(\mathrm{T}-\mathrm{H})^{-}$ & $(\mathrm{T}-2 \mathrm{H})^{-}$ & $\left(\mathrm{C}_{4} \mathrm{H}_{5} \mathrm{~N}_{2} \mathrm{O}\right)^{-}$ & $\left(\mathrm{C}_{3} \mathrm{H}_{2} \mathrm{NO}\right)^{-}$ & $\left(\mathrm{C}_{3} \mathrm{H}_{4} \mathrm{~N}\right)^{-}$ & $(\mathrm{OCN})^{-}$ & $(\mathrm{CN})^{-}$ \\
\hline 0.16 & 0.04 & 0 & & & & & \\
\hline 0.71 & 0.7 & & & & & & \\
\hline 1.02 & 1.02 & & & & & & \\
\hline 1.24 & 1.24 & & & & & & \\
\hline 1.41 & 1.47 & 1.41 & & & & & \\
\hline 1.73 & 1.74 & & & & & & \\
\hline 1.89 & & & & & & & \\
\hline & & & & & & 2.33 & \\
\hline $\begin{array}{l}2.9 \\
5.32\end{array}$ & 54 & & & & & & \\
\hline 5.66 & & & 5.95 & & & & \\
\hline 6.71 & 6.8 & & & 6.71 & & 6.81 & \\
\hline 7.02 & & & & & 7.06 & & \\
\hline 7.48 & & 7.39 & & & & & \\
\hline 7.95 & 7.8 & & 7.96 & & & 7.91 & 6.94 \\
\hline
\end{tabular}

отрицательного иона молекулы, связанного с $\pi^{*}$ и $\sigma^{*}$-молекулярными орбиталями. Этот процесс проявляется широким максимумом в сечении прилипания при энергии $1.73 \mathrm{eV}$.

Следует также отметить возможность комплементарной реакции (процесса, дополнительного к (2a)) [40]:

$$
e^{-}+\mathrm{T} \leftrightarrow(\mathrm{T})^{-*} \rightarrow(\mathrm{T}-\mathrm{H})+\mathrm{H}^{-}
$$

с образованием отрицательного иона водорода. Этот процесс также является избирательным к положению атома Н и его связи с другими атомами молекулы. Из сравнения результатов наших измерений (рис. 2 и табл. 2) с данными работы [40] можно заключить, что особенности, наблюдаемые при энергиях 5.66 и $6.71 \mathrm{eV}$, обусловлены влиянием процесса (2) с потерей $\mathrm{H}^{-}$из положений N1 и N3 соответственно.

Интересно сопоставить проявление особенностей, наблюдаемых на энергетических зависимостях ПСР (рис. 1) и полного сечения диссоциативного прилипания электронов к молекулам тимина (рис. 2). Так же как и в случае с молекулой урацила [14], в ПСР электронов на молекулах тимина отсутствует вклад от интенсивного максимума, наблюдаемого при энергии $1.02 \mathrm{eV}$ в сечении диссоциативного прилипания. Однако широкие особенности, расположенные при энергии $\sim 1.73 \mathrm{eV}$ в этих сечениях, коррелируют.

\section{Образование положительных ионов}

Энергетическая зависимость сечения образования положительных ионов (функция ионизации) тимина при столкновении с электронами приведена на рис. 3. Первый потенциал ионизации молекулы тимина $9.2 \pm 0.05 \mathrm{eV}$, определенный нами линейной экстраполяцией начального участка экспериментальной кривой (вставка на рис. 3), удовлетворительно согласуется с данными, полученными другими методами: 9.2, 9.19, $9.43,9.18 \mathrm{eV}[41,26,24,25]$ соответственно. Как видно из рис. 3, на измеренной функции ионизации молекулы тимина наблюдается рост тока регистрируемых ионов во всем исследуемом диапазоне энергий. Кроме того, на кривой заметна структура в виде изломов и небольших ступенек (указаны стрелками на рис. 3 и вставке). Отметим, что приведенное здесь сечение имеет смысл полного сечения, так как включает в себя сечения образования ионов целой молекулы и ее фрагментов, т. е. продуктов диссоциативной ионизации.

Для выделения особенностей и более точного определения их энергетического положения из измеренной функции ионизации $\left(\sigma_{\exp }^{i}\right)$ вычли вклад прямой ионизации, аппроксимированный полиномом 7-й степени $\left(\sigma_{\text {app }}^{i}\right)$. Полученный результат $\left(\sigma_{\exp }^{i}-\sigma_{\text {app }}^{i}\right)$ (кривая 1$)$ приведен на рис. 4. На этом же рисунке для сравнения приведен фотоэлектронный спектр (кривая 2), измеренный при энергии фотонов $h v=40 \mathrm{eV}$ для угла наблюдения $\theta=90^{\circ}$ [26]. На рис. 4 мы ограничились диапазоном энергий от 8.5 до $21 \mathrm{eV}$, поскольку при более высоких энергиях интерпретация структуры сечения ионизации молекулы затруднительна. Энергетические положения особенностей, наблюдаемых на кривой 1, приведены в табл. 3. Для интерпретации полученных результатов мы воспользовались данными экспериментов и расчетов по фотоионизации молекулы тимина $[25,26,42]$, которые 
Таблица 3. Энергетическое положение особенностей, наблюдаемых в полном сечении ионизации молекулы тимина электронами в области энергий от порога до $20.5 \mathrm{eV}$

\begin{tabular}{|c|c|c|c|c|c|c|}
\hline \multicolumn{2}{|c|}{ Наши результаты } & \multirow{2}{*}{$\begin{array}{c}\text { Молекулярная } \\
\text { орбиталь } \\
{[26]}\end{array}$} & \multicolumn{4}{|c|}{$\begin{array}{c}\text { Вертикальная энергия ионизации, } \\
\text { eV }\end{array}$} \\
\hline $\begin{array}{c}\text { Область } \\
\text { энергий, } \\
\text { eV }\end{array}$ & $\begin{array}{c}\text { Вертикальная } \\
\text { энергия } \\
\text { ионизации } \\
( \pm 0.05 \mathrm{eV})\end{array}$ & & $\begin{array}{c}\text { Эксперимент } \\
( \pm 0.02 \mathrm{eV})[26]\end{array}$ & $\begin{array}{c}\text { Теория } \\
\text { OVGF [26] }\end{array}$ & $\begin{array}{c}\text { Эксперимент } \\
{[25]}\end{array}$ & $\begin{array}{c}\text { Теория } \\
{[42]}\end{array}$ \\
\hline $9.0-9.7$ & 9.2 & $6 a^{\prime \prime}\left(\pi_{6}\right)$ & 9.19 & 8.85 & $\begin{array}{l}9.18 \\
\left(\pi_{1}\right)\end{array}$ & $\begin{array}{l}9.14 \\
\left(\pi_{1}\right)\end{array}$ \\
\hline $9.7-10.25$ & 9.96 & $18 a^{\prime}(\sigma \mathrm{O})$ & 10.14 & 10.46 & $\begin{array}{c}10.03 \\
\left(n_{1}\right)\end{array}$ & $\begin{array}{l}9.95 \\
(\sigma \mathrm{O})\end{array}$ \\
\hline $10.25-12.7$ & 10.45 & $5 a^{\prime \prime}\left(\pi_{5}\right)$ & 10.45 & 10.46 & $\begin{array}{c}10.39 \\
\left(\pi_{2}\right)\end{array}$ & $\begin{array}{c}10.43 \\
\left(\pi_{2}\right)\end{array}$ \\
\hline & 12.27 & $4 a^{\prime \prime}\left(\pi_{4}\right)$ & 12.27 & 12.52 & $\begin{array}{c}10.02 \\
\left(n_{2}\right) \\
12.27 \\
\left(\pi_{3}\right)\end{array}$ & $\begin{array}{c}(\sigma \mathrm{O}) \\
12.52 \\
\left(\pi_{3}\right)\end{array}$ \\
\hline $12.7-14.12$ & 13.49 & $16 a^{\prime}(\sigma)$ & 13.31 & 13.92 & & \\
\hline $14.12-15.53$ & $\begin{array}{l}14.76 \\
14.98 \\
15.05 \\
15.37\end{array}$ & $\begin{array}{l}2 a^{\prime \prime}\left(\pi_{2}\right) \\
14 a^{\prime}(\sigma)\end{array}$ & $\begin{array}{l}14.85 \\
14.90 \\
15.02\end{array}$ & 15.49 & & \\
\hline $15.53-16.19$ & $\begin{array}{l}15.92 \\
16.11\end{array}$ & $1 a^{\prime \prime}\left(\pi_{1}\right)$ & 15.75 & 16.08 & & \\
\hline $16.19-17.36$ & $\begin{array}{l}16.67 \\
16.85 \\
17.03\end{array}$ & $\begin{array}{l}12 a^{\prime}(\sigma) \\
11 a^{\prime \prime}(\sigma)\end{array}$ & 16.86 & 16.35 & & \\
\hline $17.36-18.05$ & $\begin{array}{l}17.51 \\
17.96\end{array}$ & $\begin{array}{c}11 a^{\prime}(\sigma) \\
10 a^{\prime}(\sigma) \\
9 a^{\prime}(\sigma)\end{array}$ & 18.03 & 17.37 & & \\
\hline $18.05-18.50$ & $\begin{array}{l}18.05 \\
18.23\end{array}$ & $a^{\prime \prime}\left(\pi_{1}\right)$ & & & & \\
\hline $18.5-20.5$ & 19.47 & & & & & \\
\hline
\end{tabular}

также приведены в этой таблице. Укажем, что на рис. 4 стрелками показаны расчетные данные вертикальных энергий ионизации, взятые из работы [26].

Появление тока ионов при значении энергии электронов $9.2 \mathrm{eV}$ (рис. 3) свидетельствует об образовании материнского иона $\mathrm{T}^{+}$молекулы тимина в результате прямого процесса ионизации - выбивания электрона из $\pi$-орбитали внешней оболочки молекулы [26]. Ионизация приводит к изменению длин связей - увеличению длины связи С5-С6 и уменьшению длины связи N1-C6 (см. структурную формулу молекулы на рис. 2). Так, длины связей C5-C6 и N1-C6 в нейтральной молекуле тимина равны 1.344 и $1.379 \AA$, тогда как в катионе 1.409 и $1.320 \AA[43]$.

В диапазоне энергий ионизирующих электронов от порога до $14 \mathrm{eV}$ на функции ионизации наблюдаются небольшие изломы при энергиях 9.20, 9.82, 10.23, 10.51, $10.65,11.99,13.08$ и $13.93 \mathrm{eV}$ (частично указаны стрелками на вставке и рис. 3). По аналогии с работой [44] эти значения энергий были определены как точки пересечения каждого из серии аппроксимированных линейных сегментов функции ионизации. Эти энергии соответствуют порогам (энергиям появления) новых ионизационных процессов. 
Широкая особенность с максимумом при энергии $11.5 \mathrm{eV}$, очевидно, является результатом суперпозиции нескольких полос (кривая 1 на рис. 4). Так, сравнение кривой 1 с фотоэлектронным спектром тимина (кривая 2) показывает, что эта особенность является результатом перекрывания по меньшей мере двух полос (с максимумами при 10.89 и $12.27 \mathrm{eV}$ ). Эти полосы связаны, согласно расчетам методом OVGF (outer-valence Green's function) [26], с ионизацией из $\sigma$ - и $\pi$-орбиталей внешней оболочки молекулы соответственно (табл. 3).

В области энергий $14.12-15.53 \mathrm{eV}$ наблюдается структура, интерпретировать которую однозначно очень сложно из-за присутствия множества сателлитов [26]. Энергетическое положение этой широкой особенности совпадает с широкой особенностью, наблюдаемой в фотоэлектронном спектре (кривая 2). Согласно расчетам [26], она обязана удалению $2 a^{\prime \prime}\left(\pi_{2}\right)$ - и $14 a^{\prime}(\sigma)$-электронов молекулы.

Как видно из рис. 4, значительные вклады в полное сечение ионизации наблюдаются также в области энергий выше $15.5 \mathrm{eV}$. Положения особенностей, наблюдаемых нами при энергиях $\sim 16.7$ и $\sim 18 \mathrm{eV}$, совпадают с особенностями в фотоэлектронном спектре. Согласно [26], их появление обусловлено удалением $11 a^{\prime}(\sigma)$-,

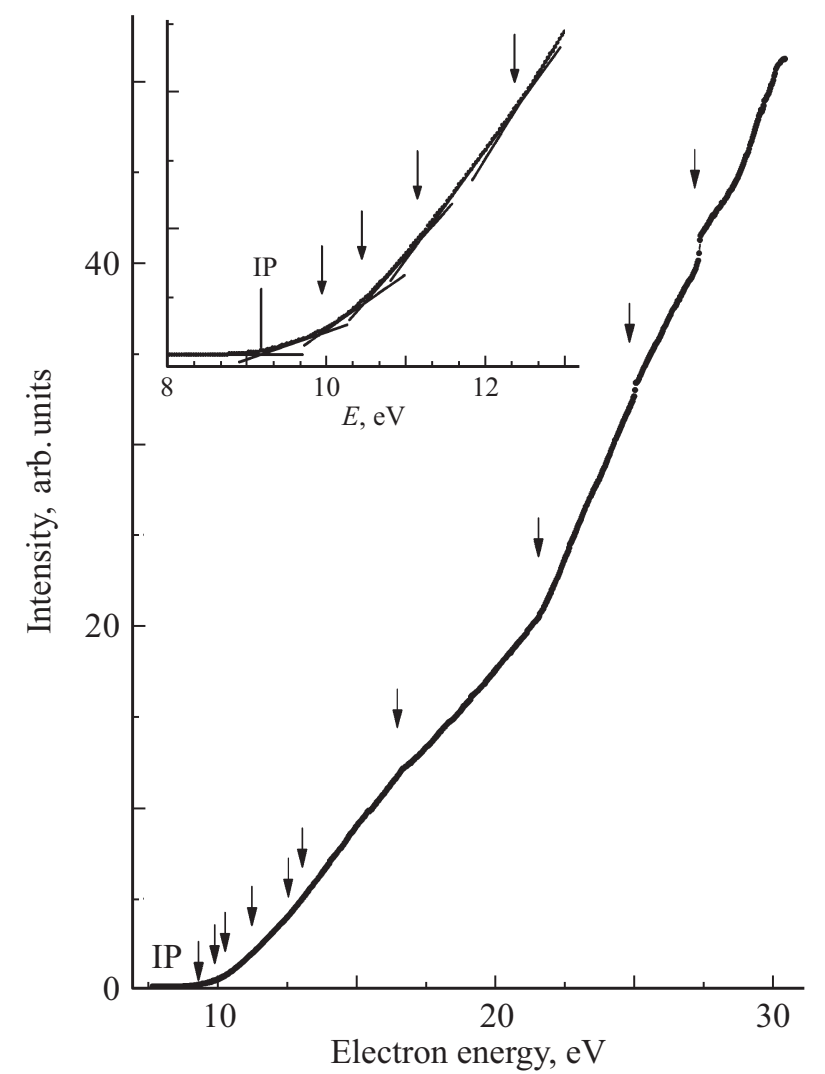

Pис. 3. Энергетическая зависимость полного сечения образования положительных ионов при столкновении электронов с молекулами тимина. На вставке показан припороговый участок сечения ионизации.

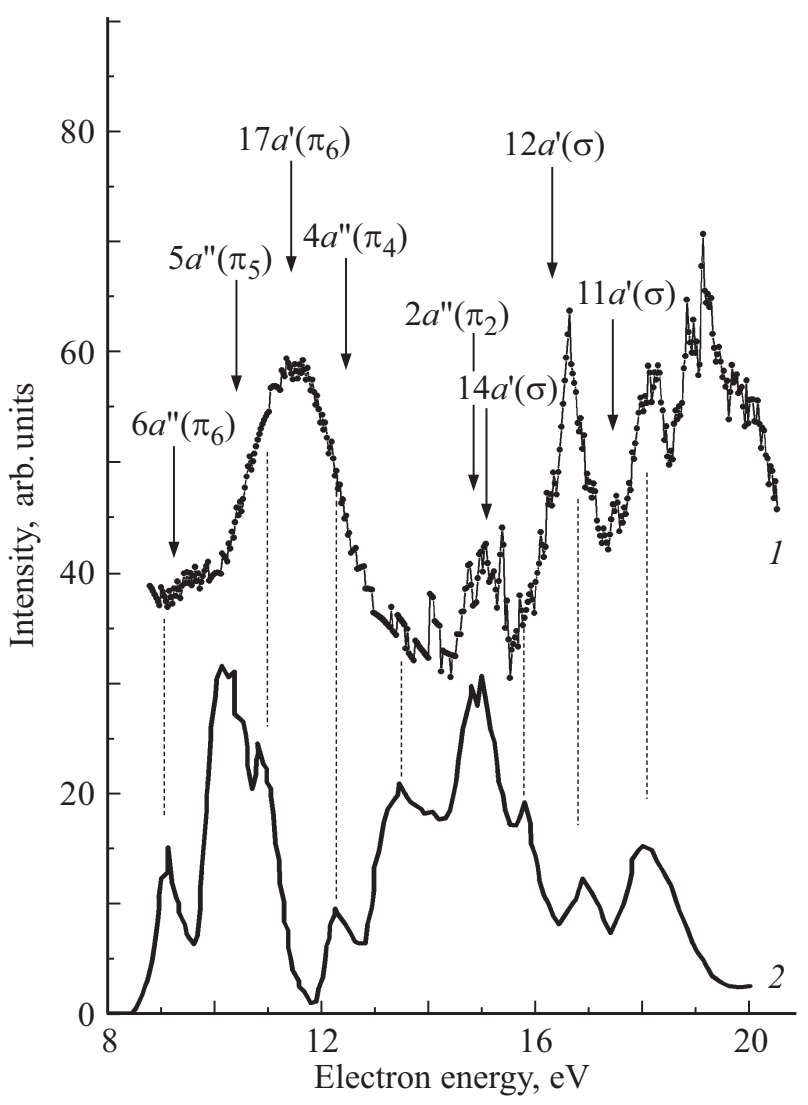

Рис. 4. Сравнение структуры, наблюдаемой в полном сечении ионизации (1) с фотоэлектронным спектром, измеренным для энергии фотонов $h v=40 \mathrm{eV}$ и угла наблюдения $\theta=90^{\circ}[26](2)$.

а также $10 a^{\prime}(\sigma)$ - и $9 a^{\prime}(\sigma)$-электронов внешней оболочки молекулы.

Анализ результатов наших измерений с использованием работ $[23,27]$, показал, что при ионизации молекулы тимина вблизи энергии $10.65 \mathrm{eV}$ наблюдается влияние процесса образования ионного фрагмента:

$$
\begin{gathered}
\mathrm{C}_{5} \mathrm{H}_{6} \mathrm{~N}_{2} \mathrm{O}_{2}+e^{-} \rightarrow \mathrm{C}_{5} \mathrm{H}_{6} \mathrm{~N}_{2} \mathrm{O}_{2}^{+}+2 e \rightarrow \mathrm{C}_{4} \mathrm{H}_{5} \mathrm{NO}^{+} \\
(A E=10.70 \mathrm{eV})+\mathrm{HNCO}+e^{-} .
\end{gathered}
$$

Этот ион образуется в результате разрыва двух связей N3-C4 и C2-N1 материнского иона. Последняя связь намного слабее в катионе, чем в нейтральной молекуле [27].

Изломы, наблюдаемые в сечении ионизации тимина (рис. 3) при больших значениях энергии электронов $21.5,25.00$ и $27.3 \mathrm{eV}$, возможно, связаны с диссоциативной ионизацией молекулы [19]. Так, в работе [20] наблюдали ионные фрагменты $m / z=51,52$ и $37 \mathrm{amu,}$ которые имеют энергии появления выше $20 \mathrm{eV}$. 


\section{Заключение}

Исследованы процессы рассеяния электронов, образования отрицательных и положительных ионов, происходящие при столкновениях медленных электронов с молекулами тимина в газовой фазе.

Проведенные исследования показали, что взаимодействие электронов с молекулами тимина в области низких энергий $(E<8 \mathrm{eV})$ носит резонансный характер. Наличие резонансов выявлено как в полном сечении рассеяния, так и в сечении диссоциативного прилипания электронов к молекулам. В полном сечении рассеяния в области энергий ниже $4.5 \mathrm{eV}$ обнаружены три резонанса формы, которые относятся к $\pi^{*}$-состояниям. Дополнительно в области энергий выше $4.5 \mathrm{eV}$ мы наблюдали еще несколько резонансных состояний, которые можно отнести к резонансам Фешбаха (core-excited-резонансы).

В полном сечении диссоциативного прилипания электронов к молекулам тимина в области энергий $E<4 \mathrm{eV}$ наблюдается четкая структура, обусловленная образованием отрицательного иона $(\mathrm{T}-\mathrm{H})^{-}$. Наши исследования показали, что отрицательные ионные фрагменты формируются и в области энергий выше $4 \mathrm{eV}$, однако эффективность их образования в $\sim 2$ раза ниже. Проанализирована корреляция особенностей, обнаруженных в двух этих сечениях. Показано, что в полном сечении рассеяния электронов отсутствует вклад от интенсивного максимума, наблюдаемого в полном сечении диссоциативного прилипания при энергии $1.02 \mathrm{eV}$, но коррелируют широкие особенности, расположенные при энергии $\sim 1.73 \mathrm{eV}$.

Продемонстрировано, что процессы образования положительных ионов молекулы тимина вблизи порога осуществляются за счет удаления электронов из $\sigma$ - и $\pi$-орбиталей валентной оболочки молекулы. Кроме того, в исследованном диапазоне энергий (от порога до $32 \mathrm{eV}$ ) наблюдается вклад процесса диссоциативной ионизации фрагментов.

\section{Список литературы}

[1] Sanche L. // Eur. Phys. J. D. 2005. V. 35. P. 367.

[2] Mc Conkey J.W., Malone C.P., Johnson P.V., Winstead C., Mc Koy V., Kanik I. // Phys. Rep. 2008. V. 466. P. 1.

[3] Boudaiffa B., Cloutier P., Hunting D., Huels M.A., Sanche L. // Science. 2000. V. 287. P. 1658.

[4] Dugal P.-C., Abdoul H., Sanche L. // J. Chem. Phys. B. 2000. V. 104. P. 5610.

[5] Huels M.A., Boudaiffa B., Cloutier P., Hunting D., Sanche L. // J. Am. Chem. Soc. 2003. V. 125. P. 4467.

[6] Aflatooni K., Gallup G.A., Burrow P.D. // J. Phys. Chem. A. 1998. V. 102. P. 6205.

[7] Abouaf R., Pommier J., Dunet H. // Int. J. Mass. Spectr. 2003. V. 226. P. 397.

[8] Abouaf R., Pommier J., Dunet H. // Chem. Phys. Lett. 2003. V. 381. P. 486.
[9] Hanel G., Gstir B., Denifl S., Scheier P., Probst M., Farizon B., Farizon M., Illenberger E., Mark T.D. // Phys. Rev. Lett. 2003. V. 90. N 18. P. 188104.

[10] Denifl S., Ptasinska S., Cingel M., Matejcik S., Scheier P., Mark T.D. // Chem. Phys. Lett. 2003. V. 377. P. 74.

[11] Шафраньош И.И., Суховия М.И., Шафраньош М.И., Шимон Л.Л. // ЖТФ. 2008. Т. 78. В. 12. С. 7.

[12] Denifl S., Ptasinska S., Hanel G., Gstir B., Scheier P., Probst M., Farizon B., Farizon M., Matejcik S., Illenberger E., Mark T.D. // Phys. Scr. 2004. V. T110. P. 252.

[13] Denifl S., Ptasinska S., Probst M., Hrusak J., Scheier P., Mark T.D. // J. Phys. Chem. A. 2004. V. 108. P. 6562.

[14] Scheer A.M., Aflatooni K., Gallup G.A., Burrow P.D. // Phys. Rev. Lett. 2004. V. 92. P. 068102.

[15] Aflatooni K., Scheer A.M., Burrow P.D. // J. Chem. Phys. 2006. V. 125. P. 054301.

[16] Burrow P.D., Gallup G.A., Scheer A.M., Denifl S., Ptasinska S., Mark T., Scheier P. // J. Chem. Phys. 2006. V. 124. P. 124310.

[17] Эрдевди Н.М., Звенигородский В.В., Шпеник О.Б., Романова Л.Г. // Опт. и спектр. 2013. Т. 114. № 1. С. 1.

[18] Шиеник О.Б., Эрдевди Н.М., Звенигородский В.В., Романова Л.Г. // ЖПС. 2013. Т. 80. № 1. С. 46.

[19] Shafranyosh I.I., Sukhoviya M.I. // Opt. Spectrosc. 2007. V. 102. N 4. P. 500.

[20] Van der Burgt P.J.M., Mahon F., Barrett G., Gradziel M.L. // Eur. Phys. J. D. 2014. V. 68. P. 151 (1-9).

[21] Bernhardt Ph., Paretzke H.G. // Int. J. Mass. Spectr. 2003. V. 223. P. 599.

[22] Mozejko P., Sanche L. // Radiat. Phys. Chem. 2005. V. 73. P. 77.

[23] Rice J.M., Dudek G.O., Barber M. // J. Am. Chem. Soc. 1965. V. 87. P. 4569.

[24] Verkin B.I., Sukodub L.F., Yanson I.K. // Dokl. Akad. Nauk SSSR. 1976. V. 228. P. 1452.

[25] Urano S., Yang X., LeBreton P. // J. Mol. Struct. 1989. V. 214. P. 315.

[26] Trofimov A.B., Schirmer J., Kobychev V.B., Potts A.W., Holland D.M.P., Karlsson L. // J. Phys. B. 2006. V. 39. P. 305.

[27] Jochims H.-W., Schwell M., Baumgartel H., Leach S. // Chem. Phys. 2005. V. 314. P. 263.

[28] Koyler C.J. Electronic Collisions with Molecules of Biological Relevance. 2011. PhD Thesis, University of Adelaide, $140 \mathrm{p}$.

[29] Чернышова И.В., Контрош Е.Э., Маркуш П.П., Шиеник О.Б. // Опт. и спектр. 2012. Т. 113. № 1. С. 7.

[30] Чернышова И.В., Контрош Е.Э., Маркуш П.П., Шиеник О.Б. // Опт. и спектр. 2013. Т. 115. № 5. С. 31.

[31] Kontros J.E., Szoter L., Chernyshova I.V., Shpenik O.B. // J. Phys. B. 2002. V. 35. P. 2195.

[32] Романюк Н.И., Шпеник О.Б., Манди Й.А., Папn Ф.Ф., Чернишова И.В. // ЖТФ. 1993. Т. 63. С. 138.

[33] NIST Chemistry webbook, available at: http://webbook.nist.gov.

[34] Winstead C., Mc Koy V. // J. Chem. Phys. 2006. V. 125. P. 174304.

[35] Dora A., Tennyson J., Bryjko L., van Mourik T. // J. Chem. Phys. 2009. V. 130. P. 164307.

[36] Sevilla M.D., Besler B., Colson A.-O. // J. Phys. Chem. 1995. V. 99. P. 1060.

[37] Winstead C., Mc Koy V., Sanchez S. // J. Chem. Phys. 2007. V. 127. P. 085105.

[38] Jordan K.D., Burrow P.D. // Chem. Rev. 1987. V. 87. P. 557. 
[39] Илленбергер Е., Смирнов Б.М. // УФН. 1998. Т. 168. № 7. C. 731.

[40] Ptasinska S., Denifl S., Grill V., Mark T.D., Illenberger Eu., Scheier P. // Phys. Rev. Lett. 2005. V. 95. P. 093201.

[41] Dougherty D., Wittel K., Meeks J., McGlynn S.P. // J. Am. Chem. Soc. 1976. V. 98. P. 3817.

[42] Dolgounitcheva O., Zakrzewski V.G., Ortiz J.V. // J. Phys. Chem. A. 2002. V. 106. P. 8411.

[43] Bravaya K.B., Kostko O., Dolgikh S., Landau A., Ahmed M., Krylov A.I. // J. Phys. Chem. A. 2010. V. 114. P. 12305.

[44] Rosinger W., Grade M., Hirschwald W. // Int. J. Mass Spect. Ion Phys. 1983. V. 47. P. 239. 